\title{
Action observation and imitation in Parkinson's disease: The influence of biological and non- biological stimuli
}

\begin{abstract}
$\underline{\text { Abstract }}$
Action observation and imitation have been found to influence movement in people with Parkinson's disease (PD), but simple visual stimuli can also guide their movement, and previous studies have not directly compared these. To investigate whether action observation may provide a more effective stimulus, the present study examined the effects of observing human pointing movements and simple visual cues on hand kinematics and eye movements in people with mild to moderate PD and age-matched controls.

In Experiment 1 participants observed videos of movement sequences between horizontal positions, depicted by a simple cue with or without a moving human hand, then imitated the sequence either without further visual input (consecutive) or while watching the video again (concurrent). Modulation of movement duration in accordance with changes in the observed stimulus increased when the simple cue was accompanied by the hand, and in the concurrent task, whereas modulation of horizontal amplitude was greater with the simple cue alone and in the consecutive task.

Experiment 2 compared imitation of kinematically-matched dynamic biological (human hand) and nonbiological (shape) stimuli, which moved with a high or low vertical trajectory. Both groups exhibited greater modulation for the hand than the shape, and differences in eye movements suggested closer tracking of the hand.

Despite producing slower and smaller movements overall, the PD group showed a similar pattern of imitation to controls across conditions. The findings demonstrate that observing human action influences aspects of movement such as duration or trajectory more strongly than non-biological stimuli, particularly during concurrent imitation.
\end{abstract}

\section{$\underline{\text { Keywords }}$}

Parkinson's disease; action observation; imitation; motor simulation; motor imagery; eye movements; kinematics; neurorehabilitation 


\section{Introduction}

Parkinson's disease (PD) is a neurodegenerative condition in which depletion of dopamine in the basal ganglia results in a range of motor impairments, impacting upon functional mobility and the ability to perform daily activities. The internal generation of movement, which relies on cortical-subcortical networks, is particularly affected in PD (Brown and Marsden, 1988; Redgrave et al., 2010). Conversely, movement can be elicited by external stimuli such as simple visual and auditory cues; for example, laser pointers or rhythmic stimuli may improve aspects of gait (Spaulding et al., 2013; van Wegen et al., 2014). However, these sensory cues are less readily applied to movements involving finer motor control, such as the manual actions needed to perform many activities of daily living.

Movement may also be influenced by action observation (AO), which activates a network of fronto-parietal neural structures partially overlapping with those involved in motor execution (Caspers et al., 2010; Hardwick et al., 2018). Imitation, which involves execution of the observed action, engages a broader brain network (Caspers et al., 2010) and has a key role in motor learning and social interaction (lacoboni et al., 2005). Studies in healthy participants have demonstrated behavioural and neural effects of $A O$ and imitative learning in various tasks including timing, sequencing and coordination of hand actions (e.g., Higuchi et al., 2012; Sakreida et al., 2018; Stefan et al., 2008; Vogt and Thomaschke, 2007).

Applications of $A O$ and imitation have been investigated in different fields including sport (e.g., Holmes and Calmels, 2008), music (e.g., Haslinger et al., 2005) and neurological rehabilitation (e.g., Buccino, 2014; Caligiore et al., 2017). The majority of previous work in the latter area has focused on stroke, with some evidence of positive effects on motor function and particularly upper limb movements, as well as activities of daily living (for review see Borges et al., 2018). While fewer studies have been conducted in PD, AO and imitation have been found to influence the timing and amplitude of hand and arm movements (Bek et al., 2019; Castiello et al., 2009; Pelosin et al., 2013; Robles-Garcia et al., 2013), and potential improvements in gait, balance, dexterity and functional independence have been indicated following AO training (Agosta et al., 2017; Buccino et al., 2011; Caligiore et al., 2013; Giorgi et al., 2018). By activating sensorimotor networks involved in motor preparation and execution, observation of others' actions could provide a powerful stimulus for movement in $\mathrm{PD}$, with the potential to facilitate a broader range of motor tasks than simple external cues. There is also some evidence of neuroplastic effects induced by AO training in both stroke (Ertelt et al., 2007) and PD (Agosta et al., 2017).

Despite these promising findings, other studies have indicated that the internal representation of actions may be altered in PD (for review see Poliakoff, 2013). Indeed, this might be expected, given the involvement of the basal ganglia in action observation (Alegre et al., 2010; Kessler et al., 2006). For example, impaired imitation has been reported for object-directed actions (Bonivento et al., 2013; Leiguarda, 2001; Leiguarda et al., 1997) and facial expressions (Simons et al., 2004), and neuroimaging has identified potential compensatory networks involving visual processing areas during motor imagery (imagined movement) in people with PD (Helmich et al., 2012; van Nuenen et al., 2012). Additionally, impaired perception of biological stimuli has been found in PD relative to age-matched controls (Jaywant et al., 2016b; Liu et al., 2017), as well as difficulties with emotion recognition and social cognition that have been linked to the action observation network (Alegre et al., 2011; Ricciardi et al., 2017). These findings suggest that AO-based cueing or training may not be more effective than simple cueing in this population.

Based on the current evidence, it is unclear whether $A O$ exerts a stronger influence on movement than simple visual cues in people with PD, since previous studies in PD typically have not directly compared the 
effects of $A O$ and simple non-biological visual stimuli. Instead, imitated movements have been compared with verbally instructed or spontaneous movements (e.g., Castiello et al., 2009; Robles-Garcia et al., 2013), so imitation effects could potentially be explained in terms of a more general visual cueing response. However, other evidence suggests the involvement of motor simulation processes during $A O$ and imitation in people with PD. In a task tapping into a more automatic form of imitation or motor resonance, people with PD have shown greater interference for incidentally-observed movements depicted by a human finger than a simple shape, indicating a preserved sensitivity to biological stimuli (Bek et al., 2018). Additionally, deliberate imitation of a moving human hand was increased when people with PD were instructed to imagine themselves performing the observed action (Bek et al., 2019). Building on these findings, further evidence is needed to understand whether the motor effects of action observation are preserved in PD.

To investigate this issue, the present study examined observation and imitation of different stimuli in participants with PD and age-matched controls in two experiments based on studies by Wild et al. (2010; 2012). In Experiment 1, imitation of sequences shown by a simple visual cue was compared in the presence versus absence of a moving human hand. Experiment 2 then compared imitation and eye movements for observed biological (human hand) versus non-biological (shape) stimuli that were matched for kinematic information. In particular, the effects of $A O$ on the timing (duration) and size (amplitude) of movements were explored, since these parameters are commonly impaired in PD. This builds on Wild et al.'s findings of imitation of duration and amplitude in young healthy participants, as well as the recent demonstration of quantitative effects of imitation on hand movement amplitude in PD (Bek et al., 2019).

Experiment 1 examined the extent to which hand kinematics were modulated when imitating movements differing in duration (faster vs. slower) and amplitude (shorter vs. longer), which were demonstrated by a simple visual cue presented either alone or accompanied by a video of a moving human hand. If action observation is a powerful movement stimulus for people with PD, the extent to which participants modulate their kinematics according to the duration and amplitude of the observed movements (i.e., imitate) may be greater when visual cues are combined with human movement than for visual cues alone. Alternatively, in accordance with the goal-directed theory of imitation (Bekkering et al., 2000) and the findings of previous research in healthy adults (e.g., Wild et al., 2010; 2012), imitation may be driven primarily by the simple cue, with human movement providing little or no further benefit.

Additionally, since previous studies in PD have differed in the timing of imitation relative to observation, two versions of the imitation task were compared. In a consecutive task, participants observed the sequence and then executed the movement without further visual input, while in a concurrent version the stimulus video was presented again during action execution. Prior observation may facilitate action selection and planning, and might also allow closer attention to the kinematics of the movement when the observer is not required to simultaneously attend to their own movement. However, concurrent observation reduces demands on working memory and provides an online input that may directly influence ongoing motor control, possibly through increasing motor simulation (e.g., Krüger et al., 2014). It was therefore expected that observing the movement prior to, and again during, imitation, would maximise the effects. Alternatively, concurrent observation and imitation may be cognitively demanding even following prior observation, because of the need to divide attention between the ongoing visual stimulus and execution of the action (see see Eaves et al., 2016). 


\section{Experiment 1}

\subsection{Method}

\subsubsection{Participants}

Participant characteristics are shown in Table 1. Twenty-nine individuals with mild to moderate idiopathic PD and with no history of surgical intervention were recruited through local neurology clinics and Parkinson's UK. Apart from one individual, all were taking dopaminergic medication and those who reported experiencing motor fluctuations were tested in the "on" state (while medication is effectively controlling symptoms). Twenty-five control participants with no history of neurological injury or illness were recruited from the local community. The study was approved by a UK National Health Service Research Ethics Committee and written informed consent was obtained from all participants. Participants had normal or corrected-to-normal vision and were screened for dementia (Hsieh et al., 2013). All participants except for two in the PD group and two in the control group were right-handed. There was no significant difference in age or sex between groups (see Table 1).

Table 1. Experiment 1: Participant characteristics.

\begin{tabular}{lllll}
\hline & PD & Control & Statistic & P value \\
\hline Age (M, SD) & $65.1(6.2)$ & $67.1(5.4)$ & $\mathrm{t}=1.82$ & .075 \\
Sex (\% female) & 34.5 & 44.0 & $\chi^{2}=.51$ & .47 \\
UPDRS-III motor score (M, SD) & $37.3(9.8)$ & $\mathrm{N} / \mathrm{A}$ & & \\
Hoehn \& Yahr stage (Mdn, IQR) & $2.00(1.0)$ & $\mathrm{N} / \mathrm{A}$ & & \\
Years since diagnosis (M, SD) & $7.4(4.3)$ & $\mathrm{N} / \mathrm{A}$ & & \\
\hline
\end{tabular}

\subsubsection{Stimuli and procedure}

The stimuli used in the imitation task are illustrated in Figure 1. Simple visual cues were presented either alone (Cue trials) or accompanied by a human hand (Action + Cue trials). In both cases, a yellow circle measuring $19 \mathrm{~mm}$ in diameter appeared in different sequences, indicating two movements between three out of four possible locations (e.g., 4-2-1) across a horizontal movement space of $450 \mathrm{~mm}$. In Action + Cue trials the videos showed a human hand visible to just beyond the wrist, with the index finger moving between the target positions, arriving at each location at the same time as the circle appeared, and departing when the circle disappeared. The hand was shown as a mirror image of the participant's hand, such that right-handed participants viewed a left hand and vice-versa. Videos depicted movements of longer $(M=273 \mathrm{~mm})$ and shorter $(M=133 \mathrm{~mm})$ horizontal amplitudes at slower $(M=990 \mathrm{~ms})$ and faster $(\mathrm{M}=670 \mathrm{~ms})$ durations.

The experiment was programmed in Presentation (Neurobehavioural Systems, Inc.), and stimuli were projected at life-size onto a $1000 \mathrm{~mm}$ x $750 \mathrm{~mm}$ screen at a distance of $1200 \mathrm{~mm}$ from the participant, who was seated at a table. A motion sensor was attached to the intermediate phalanx of the index finger of the 
dominant hand, and movements were tracked in $\mathrm{X}, \mathrm{Y}$ and $\mathrm{Z}$ axes at $120 \mathrm{~Hz}$, using a Polhemus Liberty motion tracking system with Motion Monitor software (Innovative Sports Training).

The trial sequence is illustrated in Figure 1. Trials began with a central fixation cross ( $4000 \mathrm{~ms})$, which was followed by a still image indicating the starting position for the sequence $(4000 \mathrm{~ms})$. The participant was asked to place their index finger in the equivalent starting position, mirroring the stimulus in their own workspace (on the table in front of them) and the stimulus video was then displayed (2290 - $4066 \mathrm{~ms}$ ). The action execution phase was indicated by a go-signal (green cross with "beep" sound; $1000 \mathrm{~ms}$ ), presented at a variable post-stimulus delay of 1000 - $2000 \mathrm{~ms}$. Participants then replicated the movement sequence on the table, using their dominant hand (no visual cues were present in the participant's workspace). In the concurrent task, the video was displayed again while the participant performed the action, whereas in the consecutive task, a blank screen was displayed for the same duration as the preceding video.

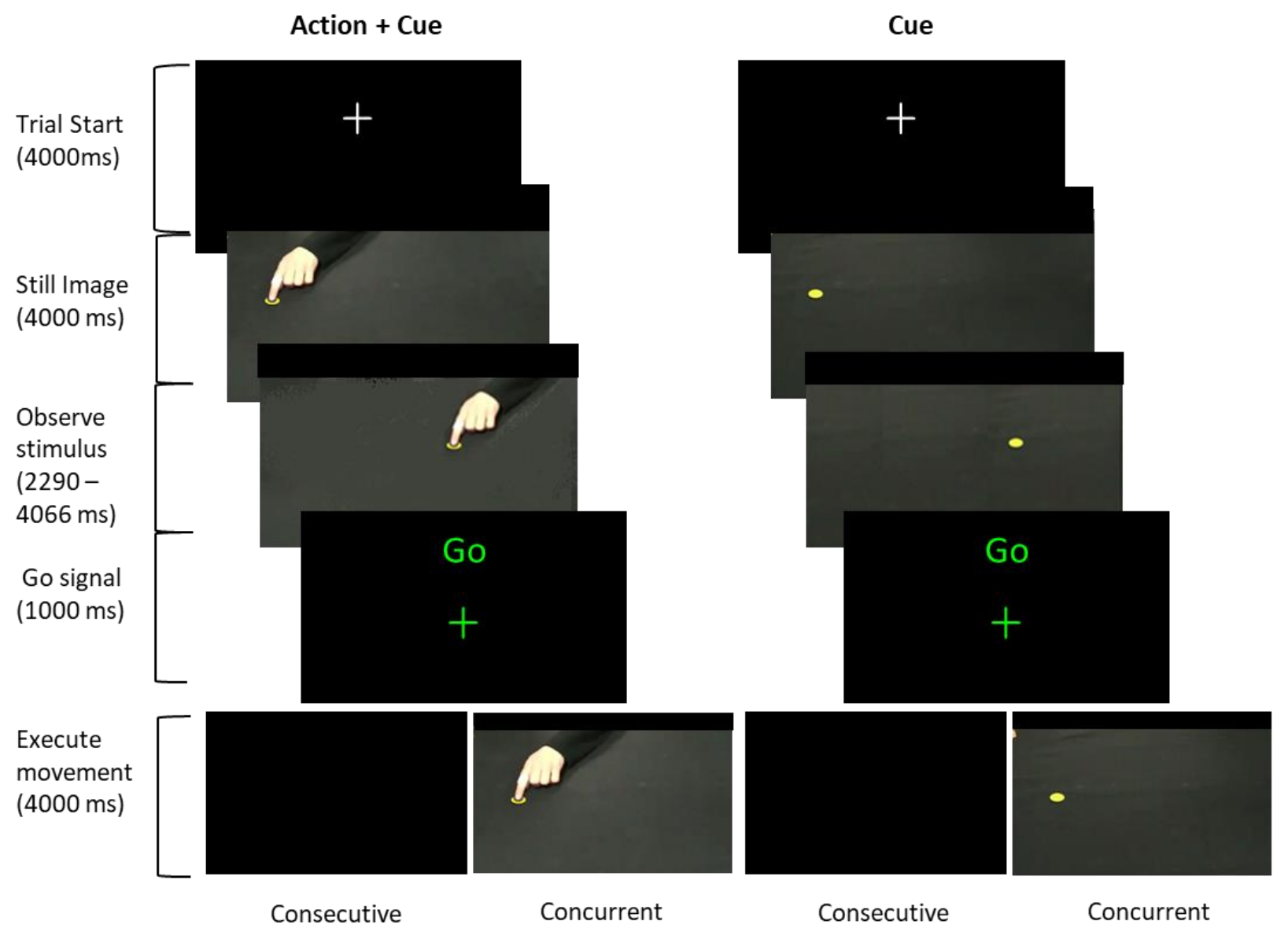

Figure 1. Imitation task in Experiment 1. Participants observed sequences illustrated by the appearance of a yellow circle (Cue) at each of 3 target locations (e.g., 4-2-1). In half of the trials, the circle was accompanied by a human hand (Action + Cue), which moved between positions, with the index finger pointing to each target location in the sequence as the circle appeared. Participants then imitated the sequence following a short delay. In the consecutive task a blank screen was presented during action execution, while in the concurrent task the video was presented for a second time. 
Participants were given the following instruction: "Please watch the video carefully and then after the beep, copy what you have seen as closely as you can in terms of the timing and distance of the movement". In the concurrent task, participants were also told: "You will see the video again while you are making the movement".

Consecutive and concurrent versions of the task were presented in two separate blocks, the order of which was counterbalanced between participants. Following a set of 4 practice trials, each test block contained 32 trials (16 Cue, 16 Action + Cue; with equal numbers of longer/shorter and faster/slower movements across conditions), presented in a randomised order. A pause halfway through each block allowed participants to take a short break.

\subsubsection{Data processing and statistical analysis}

Kinematic data from correctly-executed movements (i.e., where the correct sequence was performed) were analysed using Matlab (Mathworks Inc.). Raw data were filtered with a $20 \mathrm{~Hz}$ Butterworth filter, and movement onset and offset times were determined when velocity rose above or fell below $10 \%$ of the peak velocity for six consecutive samples. For each participant, mean movement duration (for slower vs. faster movements) and horizontal amplitude (for longer vs. shorter movements) were calculated for each stimulus condition (Cue; Action + Cue) in each task (Consecutive; Concurrent). For each trial, one movement of the two-part sequence was analysed (e.g., the 4-2 movement from the sequence 4-2-1).

All trials were screened for outliers based on the non-recursive procedure recommended by Van Selst and Jolicoeur ( 1994) at the individual level for each variable, for each stimulus type in each block. Values were removed from between $3.2 \%$ and $5.1 \%$ trials for each kinematic parameter (duration/amplitude), with similar percentages across concurrent and consecutive tasks and in each group. Across both tasks, the maximum number of trials excluded for one participant was 5 (7.8\%) in the PD group and $6(9.4 \%)$ in the control group. Inspection of between-participant outliers was then performed for each stimulus type in each block, resulting in the exclusion of data from one participant from each group in the consecutive block, and 3 from each group in the concurrent block.

Effects of the different stimuli and task versions on movement duration and amplitude were analysed using a Group (PD vs. control) x Stimulus (Cue vs. Action + Cue) x Task (Consecutive vs. Concurrent) x Duration (slower vs. faster) or Amplitude (longer vs. shorter) ANOVA. For the Duration analysis longer and shorter trials were pooled, and for the Amplitude analysis slower and faster trials were pooled. The main statistical analysis was conducted using SPSS (version 25; IBM). The accepted significance level was $p<.05$, adjusted for multiple comparisons in t-tests. Bayes Factors were also calculated using JASP (version 0.12.2.0; JASP team, 2020) to evaluate the strength of evidence for effects. A Bayes factor $\left(\mathrm{BF}_{10}\right)$ below 1 indicates increasing evidence for the null hypothesis, whereas values above 1 suggest increasing evidence for the alternative hypothesis. For interaction terms, the Bayes inclusion factor across matched models $\left(\mathrm{BF}_{\text {incl }}\right)$ is reported, which can be interpreted similarly to $\mathrm{BF}_{10}$ (Wagenmakers et al., 2018).

\subsection{Results}

Kinematic modulation effects for duration (slower - faster) and horizontal amplitude (longer - shorter) are illustrated in Figure 2. A full table of results is provided in the supplementary material (S1). 
For movement duration, there was a significant effect of Duration $\left(F(1,52)=192.63 ; p<.001 ; \eta^{2} p=.79 ; \mathrm{BF}_{10}\right.$ $=1.75 \mathrm{e}+20)$, indicating modulation in response to slower $(M=749 \mathrm{~ms})$ vs. faster $(M=668 \mathrm{~ms})$ movements. There was a significant effect of Stimulus $\left(F(1,52)=27.43 ; p<.001 ; \eta^{2} p=.35 ; B F_{10}=62.37\right)$, indicating faster movements in the Cue condition ( $M=686 \mathrm{~ms}$ ) than the Action + Cue condition ( $M=718 \mathrm{~ms}$ ). There was also an interaction between Duration and Stimulus $\left(F(1,52)=39.48 ; p<.001 ; \eta^{2} p=.43 ; \mathrm{BF}_{\text {incl }}=30.94\right)$ : modulation of movement duration was greater in the Action + Cue condition than the Cue condition ( $\mathrm{M}=$ $104 \mathrm{~ms}$ vs. $94 \mathrm{~ms} ; \mathrm{t}(53)=6.33 ; \mathrm{p}<.001)$, although significant modulation occurred in both conditions (Action + Cue, $\mathrm{t}(77)=14.12 ; \mathrm{p}<.001 ;$ Cue, $\mathrm{t}(77)=7.60 ; \mathrm{p}<.001)$. There was a non-significant effect of Task $\left(\mathrm{F}(1,52)=3.84 ; \mathrm{p}=.055 ; \eta^{2} p=.069 ; \mathrm{BF}_{10}=13.43\right)$ and a significant Task $\times$ Duration interaction $(\mathrm{F} 1,52)=$ 87.27; $\left.\mathrm{p}<.001 ; \eta^{2} p=.63 ; \mathrm{BF}_{\text {incl }}=1.46 \mathrm{e}+6\right)$, indicating greater modulation in the Concurrent task than the consecutive task $(M=121 \mathrm{~ms}$ vs. $40 \mathrm{~ms} ; \mathrm{t}(53)=8.96 ; \mathrm{p}<.001)$. However, the difference in duration when imitating faster vs. slower movements was significant for both the Concurrent task $(t(77)=16.02 ; p<.001)$ and the Consecutive task $(t(77)=5.69 ; \mathrm{p}<.001)$. There was also a significant Task $\mathrm{x}$ Stimulus interaction $\left(F(1,52)=5.24 ; p=.026 ; \eta^{2} p=.092 ; \mathrm{BF}_{\text {incl }}=.054\right)$, indicating a larger difference in duration between stimuli (Cue < Action + Cue) in the Consecutive task than the Concurrent task $(\mathrm{M}=.040 \mathrm{~ms}$ vs. $.018 \mathrm{~ms} ; \mathrm{t}(53)=$ 2.28; $p=.026)$; although the low Bayes factor suggested a weak effect, and the difference was significant for both tasks: Consecutive, $\mathrm{t}(90)=5.67 ; \mathrm{p}<.001$; Concurrent, $\mathrm{t}(90)=2.96 ; \mathrm{p}=.024$. There was a significant effect of Group on movement duration $\left(F(1,52)=25.87 ; p<.001 ; \eta^{2} p=.33 ; \mathrm{BF}_{10}=5267.06\right)$, whereby the control group exhibited faster movements overall ( $M=618 \mathrm{~ms}$ ) than the PD group ( $M=786 \mathrm{~ms}$ ).

For horizontal amplitude, there was a significant effect of Amplitude $\left(F(1,51)=1709.54 ; p<.001 ; \eta^{2} p=.97\right.$; $\left.\mathrm{BF}_{10}=2.42 \mathrm{e}+211\right)$, reflecting modulation in response to longer $(\mathrm{M}=248.94 \mathrm{~mm})$ vs. shorter $(\mathrm{M}=137.01$ $\mathrm{mm})$ movements. There was no main effect of Stimulus $\left(\mathrm{F}(1,51)=.098 ; \mathrm{p}=.76 ; \eta^{2} p=.002 ; \mathrm{BF}_{10}=.11\right)$, but there was a significant Amplitude $x$ Stimulus interaction $\left(\mathrm{F}(1,51)=11.13 ; \mathrm{p}=.002 ; \eta^{2} p=.18 ; \mathrm{BF}_{\text {incl }}=1.18\right)$, whereby modulation (longer - shorter) was greater in the Cue than Action + Cue condition ( $M=115.06 \mathrm{~mm}$ vs. $108.81 \mathrm{~ms} ; \mathrm{t}(53)=3.43 ; \mathrm{p}=.001)$. However, significant modulation was found for both $\mathrm{Cue}(\mathrm{t}(54)=$ 40.59; $p=<.001)$ and Action + Cue $(t(54)=38.69 ; p<.001)$. There was a significant effect of Task $(F(1,51)=$ $\left.19.80 ; \mathrm{p}<.001 ; \eta^{2} p=.28 ; \mathrm{BF}_{10}=.31\right)$, with larger movements in the Consecutive task $(\mathrm{M}=197.39 \mathrm{~mm})$ than the Concurrent task $(M=188.56 \mathrm{~mm})$, but the Bayes factor suggests a low strength of evidence for this effect. However, a Task $x$ Amplitude interaction $\left(F(1,51)=22.35 ; p<.001 ; \eta^{2} p=.31 ; \mathrm{BF}_{\text {incl }}=80.20\right)$, indicated greater modulation of amplitude in the Consecutive task than the Concurrent task $(M=117.49 \mathrm{~mm}$ vs. $106.38 \mathrm{~mm} ; \mathrm{t}(53)=4.84 ; \mathrm{p}<.001)$; although again significant modulation was found in both the Consecutive task $(\mathrm{t}(63)=39.46 ; \mathrm{p}=<.001)$ and the Concurrent task $(\mathrm{t}(63)=36.38 ; \mathrm{p}<.001)$. There was a significant effect of Group on amplitude $\left(\mathrm{F}(1,51)=5.58 ; \mathrm{p}=.022 ; \eta^{2} p=.099 ; \mathrm{BF}_{10}=1.11\right)$, with larger movements overall in the control group than the PD group $(M=201.79 \mathrm{~mm}$ vs. $184.16 \mathrm{~mm})$. There was also a Group $\times$ Stimulus interaction $\left(F(1,51)=6.54 ; \mathrm{p}=.014 ; \eta^{2} p=.11 ; \mathrm{BF}_{\text {incl }}=.52\right)$, reflecting a greater difference between groups (PD < Control) for Cue trials than Action + Cue trials, although the low Bayes factor suggests poor evidence in favour of this difference, and neither comparison reached significance at the corrected threshold of $\mathrm{p}<.025$ (Cue, $\mathrm{t}(49)=2.95 ; \mathrm{p}=.029$; Action + Cue, $\mathrm{t}(49)=2.36 ; \mathrm{p}=.13$ ).

There were no other significant main effects or interactions (see supplementary material S1). 


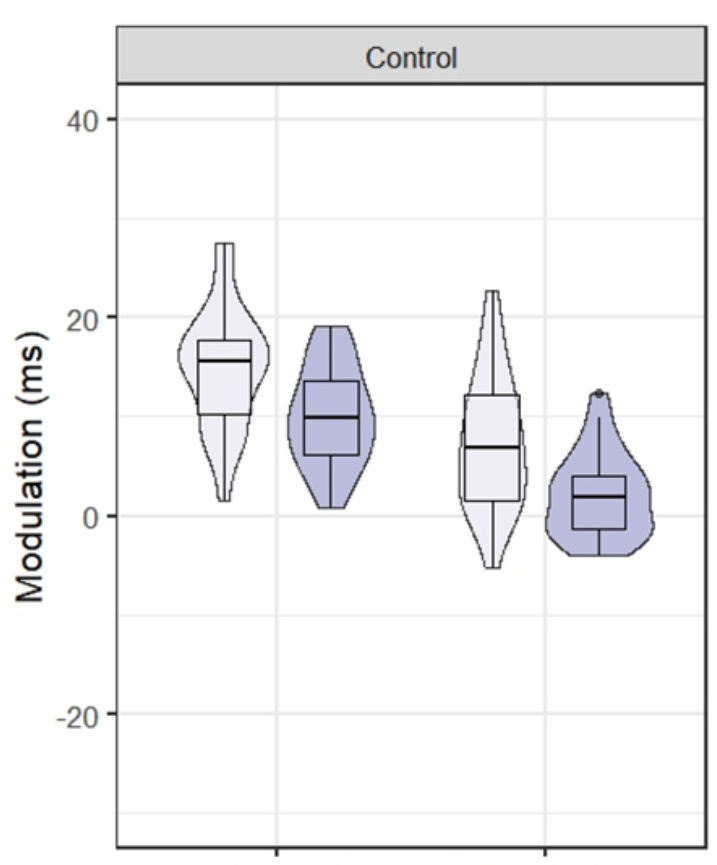

Concurrent

Consecutive
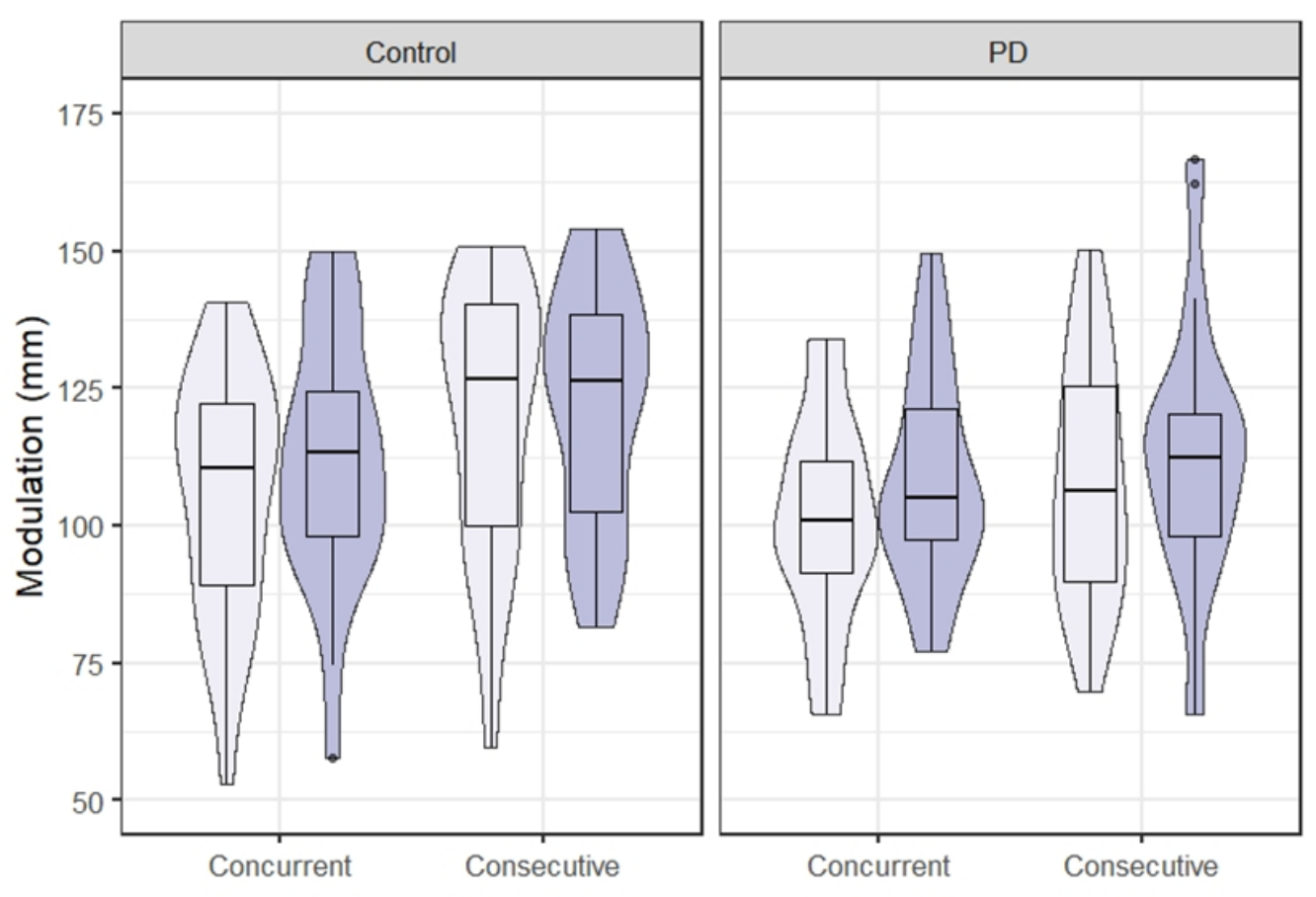

Stimulus
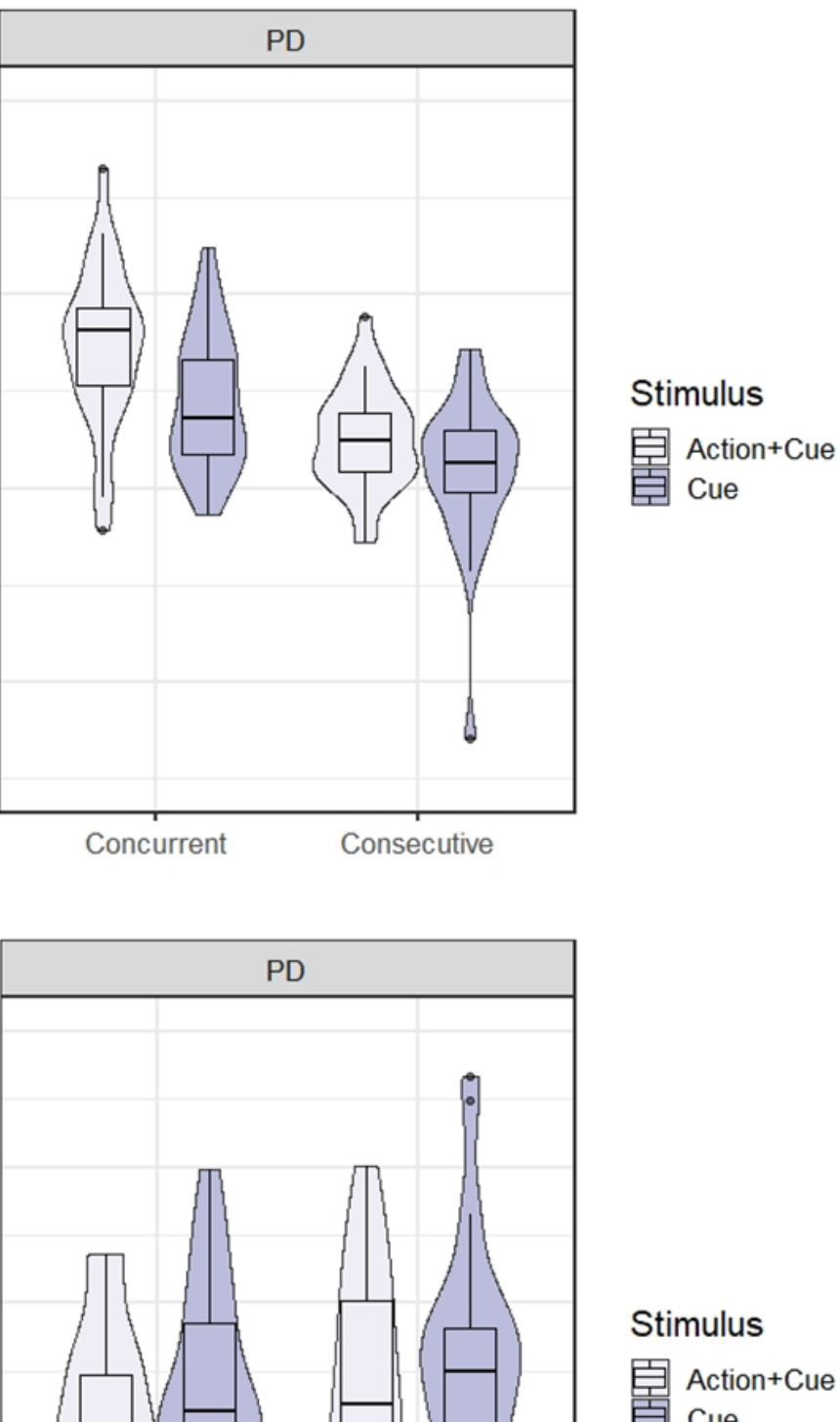

Stimulus

官 Action+Cue

Cue

Figure 2. Imitation of duration (top) and amplitude (bottom) after observing a simple cue either alone (Cue) or accompanied by a moving human hand (Action + Cue). Two versions of the imitation task were completed, in which the movement was executed either without further visual input (Consecutive task) or while the video was presented for a second time (Concurrent task). The graphs show modulation of kinematics in response to changes in the duration (slower - faster) or horizontal amplitude (longer shorter) of the observed movements. 


\subsection{Discussion}

In Experiment 1 participants imitated the duration and horizontal amplitude of sequences demonstrated by a simple visual cue that was presented either alone or combined with a moving human hand.

Although both participants with PD and age-matched controls made faster (shorter duration) movements overall after observing the simple cue alone, movement duration was modulated to a greater extent when the cue was accompanied by the hand, indicating a stronger imitation effect. Modulation of duration was also increased in the concurrent version of the task, when the stimulus was displayed again during action execution. This may reflect a reduction in demands on working memory, or increased motor simulation with the online visual input (e.g., Krüger et al., 2014). While PD participants exhibited significantly slower movements than controls, the pattern of imitation effects was the same for both groups.

In contrast to the effects on duration, modulation of horizontal amplitude was greater when the simple cue was observed alone, and modulation was increased in the consecutive task in which the stimulus video was not visible during action execution. There was some evidence that the PD group produced smaller movements overall, but again they showed a similar pattern of imitation to controls.

The differential effects of cue type on modulation of amplitude and duration might be explained as follows: when the hand was visible, participants may have attended more closely to the dynamics rather than the endpoints of the movements, whereas in the absence of the kinematic information provided by the hand, the endpoint positions (and distances between them) indicated by the simple cue were more salient. According to the goal-directed theory of imitation (Bekkering et al., 2000), imitation of kinematics should be reduced when a visible target for the movement is present. Although the present study did not directly compare imitation of a human hand movement with and without simple cues, in previous research with healthy participants the presence of visible movement endpoints has been found to reduce imitation of movement duration, but not amplitude (Wild et al., 2012, 2010). These findings together suggest that different visual stimuli may draw attention to different movement characteristics.

With respect to the difference between consecutive and concurrent tasks, regardless of cue type, the relative difference in amplitude between shorter and longer movements may have been more noticeable than the difference in duration between faster and slower trials. In the consecutive task, which relied on working memory, participants may have attended more to the endpoint positions in order to subsequently replicate the sequence correctly. In contrast, in the concurrent task, attention to the kinematics of the movement - and particularly the temporal characteristics - may have been increased while participants were not required to remember the sequence. A previous study using fMRI to compare concurrent and consecutive imitation of hand movements in healthy participants (Krüger et al., 2014) found similar accuracy in both conditions, but with different corresponding neural activations in regions related to perception and action. During concurrent imitation, accuracy was associated with activation in the left inferior parietal lobule, indicating ongoing sensorimotor mapping between the observer's own movement and the observed stimulus, while in consecutive imitation accuracy was associated with activation of the right ventral premotor cortex, suggesting comparison with stored motor representations. Although this study used a "pure" concurrent imitation condition (whereas ours included prior observation) and a different kinematic measure (angle of flexion-extension), our findings are compatible with these proposed mechanisms.

In summary, the results of Experiment 1 suggest that imitation is influenced by both the choice of stimulus and the nature of the task. Simple visual cues may be sufficient to guide movement amplitude, whereas 
human action observation may provide a more effective stimulus for movement duration, particularly when presented during execution of the action.

Given the evidence indicating compensatory processes in action representation in people with PD (Helmich et al., 2007; van Nuenen et al., 2012), it is possible that there is a greater reliance on low-level visual cues where these provide sufficient information for a particular aspect of movement, such as horizontal amplitude indicated by the distance between target locations in this experiment. However, the stronger effect of action observation on movement duration in both groups indicates that people with PD do not solely rely on simple visual cues, when additional action-relevant information is available. Nonetheless, this effect may be related to more basic visual processing of the dynamic stimulus that was provided by the human movement but not the simple cue, rather than reflecting action simulation mechanisms. Therefore, Experiment 2 investigated whether observing human movement exerts a stronger effect than nonbiological movement, when the stimuli provide equivalent dynamic information.

Evidence from action perception research suggests that biological kinematics and appearance may both be important factors in imitation. For example, in one study (McDonough et al., 2019), healthy participants exhibited a perceptual bias when observing a human hand reaching towards an object, using a straight or arched trajectory in the presence or absence of an obstacle. The hand disappeared partway through the movement and participants reported its last-seen position, showing an under- or over-estimation of the trajectory towards the most efficient path (i.e., arched when an obstacle was present, straight when it was absent). This bias was reduced when the same trajectory was shown by a non-biological, and therefore ostensibly non-agentive, stimulus. Additionally, while visuomotor priming has been found for both biological and non-biological stimuli, effects were found more often or were stronger when movements with biological kinematics were made by a biological stimulus (for review see Gowen and Poliakoff, 2012). Moreover, increased muscle activity (EMG response) has been found during observation and voluntary imitation of human-like than robotic movements (Hofree et al., 2015), as well as neural activations in the motor system corresponding to visuomotor priming effects when observing a human finger movement but not when observing a matched non-biological (dot) stimulus (Crescentini et al., 2011). In contrast, Craighero et al. (2016) found similar modulation of corticospinal excitability when observing a point-light display of a hand moving with either biological kinematics or constant velocity.

Previous studies have not directly compared the effects of matched biological and non-biological cues on imitation in PD. One laboratory-based study (Bienkiewicz et al., 2013) found that people with PD were able to imitate the speed of a moving point-light display depicting human kinematics, but this was not compared with a movement with a human appearance. In an intervention study that attempted to control for general effects of motion (Jaywant et al., 2016a), participants receiving training with video-based AO reported improved mobility compared to a control group who observed videos of moving water. However, this finding does not clearly demonstrate the importance of human movement for AO-based therapy, since the motion of water does not provide a close match for kinematic characteristics.

Experiment 2 directly compared imitation of movements with matched biological kinematic profiles that were demonstrated by either a biological agent (human hand) or a non-biological stimulus (simple shape). Although Experiment 1 showed differential effects of concurrent versus consecutive observation on imitation, a consecutive task was used in Experiment 2, which more closely reflects real-life imitative learning scenarios. In this experiment, imitation of the observed movement was examined by analysing modulation of vertical amplitude for elevated versus direct trajectories: to imitate this aspect of the movement, the observer must attend to the dynamic information provided by the stimulus, rather than just 
the endpoint. Eye gaze during observation was also recorded to further clarify neurocognitive mechanisms such as visual attention when imitating the different stimuli.

If biological and non-biological stimuli are processed differently, with greater involvement of the sensorimotor system in the former, increased imitation would be expected when movements are shown by a moving human hand than a moving shape, even when the kinematics are matched. This may be accompanied by differences in eye gaze, such as fewer eye movements and smaller saccades when observing the hand, as well as earlier fixations at the movement endpoint, indicating closer visual attention. For example, Wild et al. (2012) found increased visual tracking (fewer saccades and fixations) alongside increased imitation when observing a human hand movement in the absence of visual goals. If representation of biological movement is altered in people with PD, however, they may not exhibit these differences in imitation and eye movement for the hand compared with the shape.

\section{Experiment 2}

\subsection{Method}

\subsubsection{Participants}

Twenty-one individuals with mild to moderate idiopathic PD and 23 healthy control participants were recruited and screened as described in Experiment 1 (see Table 2 for participant characteristics). There were no significant differences between the groups in sex or age. All participants except two in the PD group were right-handed. Some of the participants (15 in each group) had also taken part in Experiment 1; however, the two studies were separated by more than a year, and participants were not aware of the specific hypotheses regarding the different stimuli. A separate analysis confirmed that there were no significant differences between returning and new participants on any of the dependent variables.

\subsubsection{Stimuli and procedure}

The stimuli used in Experiment 2 are illustrated in Figure 3. As in Experiment 1, participants observed and imitated movement sequences between 3 of 4 horizontal locations spaced $150 \mathrm{~mm}$ apart. This time, the movements were demonstrated by either a human hand (Hand condition) with the index finger pointing to each target location, or a blue square (Shape condition) of approximately the same area as the fingernail in the Hand videos $(8 \mathrm{~mm})$. The Shape stimulus was created by matching the position of the square to the coordinates of the fingertip on a frame-by-frame basis, such that both moved with the same biological kinematic profile.

In this experiment, the trajectory with which the stimulus moved between points was manipulated: elevated movements followed an indirect, curved trajectory between target positions, with a mean vertical amplitude of $131 \mathrm{~mm}$; direct movements followed a lower, non-elevated trajectory between positions, with a mean vertical amplitude of $22 \mathrm{~mm}$. Imitation of the trajectory was analysed in terms of modulation of the vertical amplitude of the participant's index finger movement when executing the observed sequence.

Stimuli were projected at life-size onto a $530 \mathrm{~mm} \times 300 \mathrm{~mm}$ screen at a distance of $700 \mathrm{~mm}$ from the participant. Kinematics were recorded using a Polhemus Fastrak electromagnetic motion capture system at a sampling rate of $120 \mathrm{~Hz}$. Eye gaze was recorded using an Eyelink 1000 Plus eye tracker (SR Research Ltd.), 
using remote monocular pupil capture at a sampling rate of $500 \mathrm{~Hz}$ and spatial resolution of $0.1^{\circ}$, with typical accuracy of $0.25-0.50$. An initial nine-point calibration was performed with each participant.

The trial sequence and timing were the same as in the consecutive task of Experiment 1 (Figure 1). Hand and Shape trials were presented in separate blocks and all participants completed the Shape block first. This design was intended to avoid potential carry-over effects, whereby the moving shape may be imagined as a human hand after prior exposure to the Hand stimulus, since previous research has shown that topdown factors such as belief about agency can influence imitation (Gowen and Poliakoff, 2012). Each block consisted of a short practice section followed by 30 test trials containing movements between the fourth and second locations (e.g., 4-2 in the sequence 4-2-1), including 10 elevated and 10 direct trials ${ }^{1}$, which were included in the analysis. The test trials were interspersed with 9 filler trials containing different sequences to reduce predictability. Trials were randomised within blocks, and there was a short pause halfway through each block. Participants were given the following instruction: "Please watch the video carefully and then after the beep, copy what you have seen as closely as you can in terms of the timing and size of the movement".

Table 2. Experiment 2: Participant characteristics.

\begin{tabular}{lllll}
\hline & PD & Control & Statistic & P value \\
\hline Age (M, SD) & $64.7(7.0)$ & $68.0(7.3)$ & $\mathrm{t}=1.56$ & .13 \\
Sex (\% female) & 27.3 & 43.5 & $\chi^{2}=1.29$ & .26 \\
UPDRS-III motor score (M, SD) & $38.4(11.3)$ & $\mathrm{N} / \mathrm{A}$ & & \\
Hoehn \& Yahr (Mdn, IQR) & $2.00(1.5)$ & $\mathrm{N} / \mathrm{A}$ & \\
Years since diagnosis (M, SD) & $6.8(4.8)$ & $\mathrm{N} / \mathrm{A}$ & \\
\end{tabular}
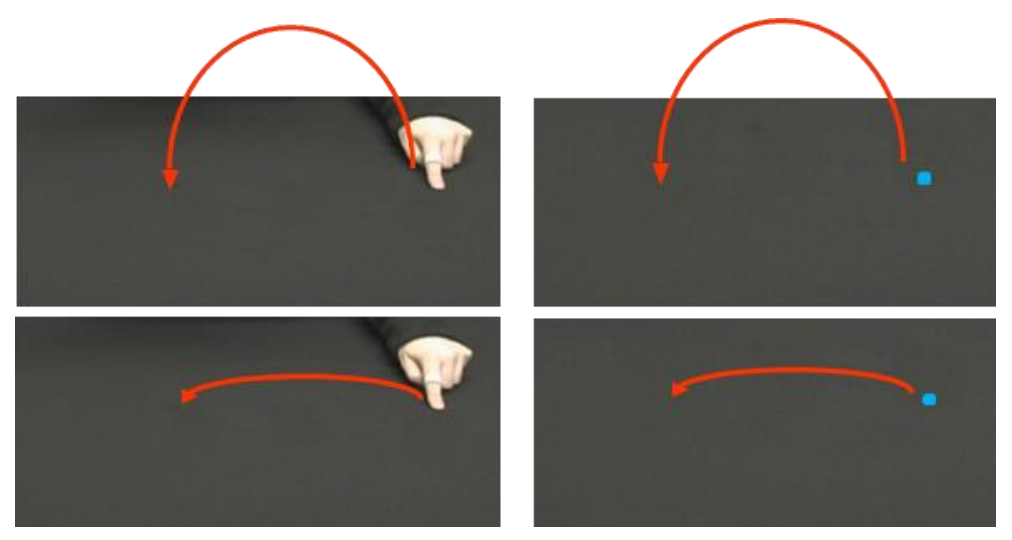

Figure 3. Imitation stimuli in Experiment 2: Movements followed an elevated (top) or direct (bottom) biological trajectory, and were demonstrated by either a human hand (left) or simple shape (right).

\footnotetext{
${ }^{1} 10$ trials in each block showed slightly faster direct movements, which were included to explore potential imitation effects for timing. However, the difference in duration was very subtle and there was no significant modulation effect, so these trials were omitted from further analysis.
} 


\subsubsection{Data processing and statistical analysis}

Kinematic data were unavailable for one participant in the PD group because of recording error. Kinematics of correctly-executed 4-2 movements were extracted as in Experiment 1 and mean vertical amplitude was calculated for Hand and Shape blocks. Outliers were identified and removed as described above, resulting in the exclusion of data from $1.1 \%$ trials in the Hand block and $1.0 \%$ trials- in the Shape block in the PD group, and $0.3 \%$ in the Hand block and $0.6 \%$ in the Shape block in the control group. The maximum number of trials excluded per participant across conditions was $2(3.3 \%)$ in the PD group and $1(1.7 \%)$ in the control group. At the between-participant level, outliers were calculated for each group within each block. Data from one participant in the control group were excluded for both Hand and Shape blocks. Kinematic data were then analysed using a Group (PD vs. control) x Stimulus (Hand vs. Shape) x Trajectory (elevated vs. direct) ANOVA.

Because of calibration difficulties and loss of data (e.g., due to excess blinking), reliable eye movement recordings were only available for 15 participants in the PD group and 20 participants in the control group. Eye tracking data were extracted and summarised using Data Viewer (Version 2.4.0.115; SR Research Ltd.) using the default settings, with no minimum fixation duration specified. Trials were then inspected further for loss of data, resulting in the removal of $1.3 \%$ trials across blocks in the PD group and $2.4 \%$ trials in the control group. In addition to analysing general eye movement characteristics (fixations and saccades), an interest area was created at $\pm 30 \mathrm{~mm}$ around the second target location, which was the endpoint of the 4-2 movement (see Figure 4). For each participant, the number and mean duration of fixations, and the number and mean amplitude of saccades, were calculated for each trial, as well as the time of the first fixation in the interest area.

Outliers were identified for each variable at the individual level. In the PD group, this resulted in the loss of $9.9 \%$ values from the Hand block and $4.8 \%$ from the Shape block; in the control group $9.1 \%$ values were excluded from the Hand block and $10.2 \%$ from the Shape block, with a maximum of $5(16.7 \%)$ trials per participant excluded from each block in each group. Outliers were also identified at the betweenparticipant level for each variable. In the PD group, data were excluded from 3 participants in the Hand block (one for fixation duration, two for first fixation in the interest area) and one in the Shape block (fixation duration). In the control group data were excluded from 3 participants in the Hand block (one for fixation count and saccade count, 2 for first fixation in the interest area) and 3 in the Shape block (one for fixation duration, 2 for first fixation in the interest area). Eye movement variables were then analysed using Group (PD vs. control) $x$ Stimulus (Hand vs. Shape) ANOVAs.

Interaction effects were explored using Bonferroni-corrected pairwise comparisons, and Bayes factors were calculated to further examine the strength of evidence for effects.

To ensure that any differences between the two conditions (Hand and Shape) were not an artefact of practice effects arising from the fixed order of the blocks, we conducted additional analyses comparing kinematic and eye movement data between the first and second half of each block. These analyses indicated no difference between conditions in practice effects for eye movements, and greater evidence of increased imitation between the Shape and Hand blocks than within the Shape block (see supplementary material S2 for detailed results). 


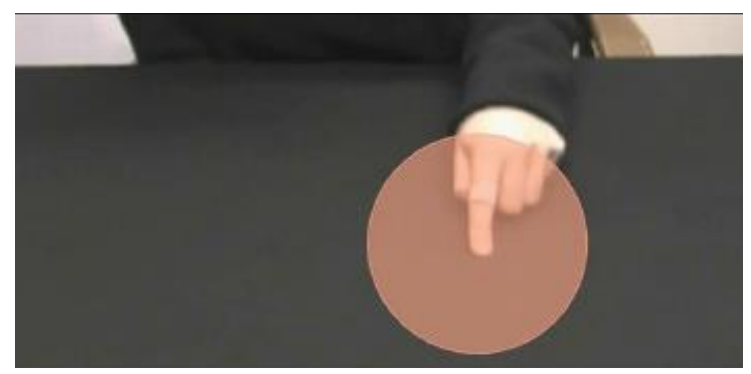

Figure 4. In Experiment 2 an interest area was created to examine the timing of fixations around the movement endpoint.

\subsection{Results}

\subsubsection{Kinematics}

Hand movement kinematics are presented in Figure 5. There were significant effects of Trajectory $(F(1,40)=$ 103.48; $\left.\mathrm{p}<.001 ; \eta^{2} p=.72 ; \mathrm{BF}_{10}=1.18 \mathrm{e}+22\right)$ and Stimulus $\left(\mathrm{F}(1,40)=21.34 ; \mathrm{p}<.001 ; \eta^{2} p=.35 ; \mathrm{BF}_{10}=2.06\right)$ on the vertical amplitude of hand movements, reflecting larger movements in elevated $(M=120.0 \mathrm{~mm})$ than direct $(M=42.55 \mathrm{~mm})$ trials, and in the Hand block $(M=91.24 \mathrm{~mm}$ ) compared to the Shape block ( $M$ $=70.47 \mathrm{~mm})$. There was also a significant interaction between Stimulus and Trajectory $(F(1,40)=26.73 ; p$ $\left.<.001 ; \eta^{2} p=.40 ; \mathrm{BF}_{\text {incl }}=26.03\right)$, indicating greater modulation of vertical amplitude in the Hand block $(\mathrm{M}=$ $95.21 \mathrm{~mm})$ than the Shape block $(M=59.21 \mathrm{~mm}) ; \mathrm{t}(41)=5.21 ; \mathrm{p}<.001$. Nonetheless, modulation was significant in both the Hand $(t(60)=12.15 ; p<.001)$ and Shape $(t(60)=7.05 ; p<.001)$ blocks.
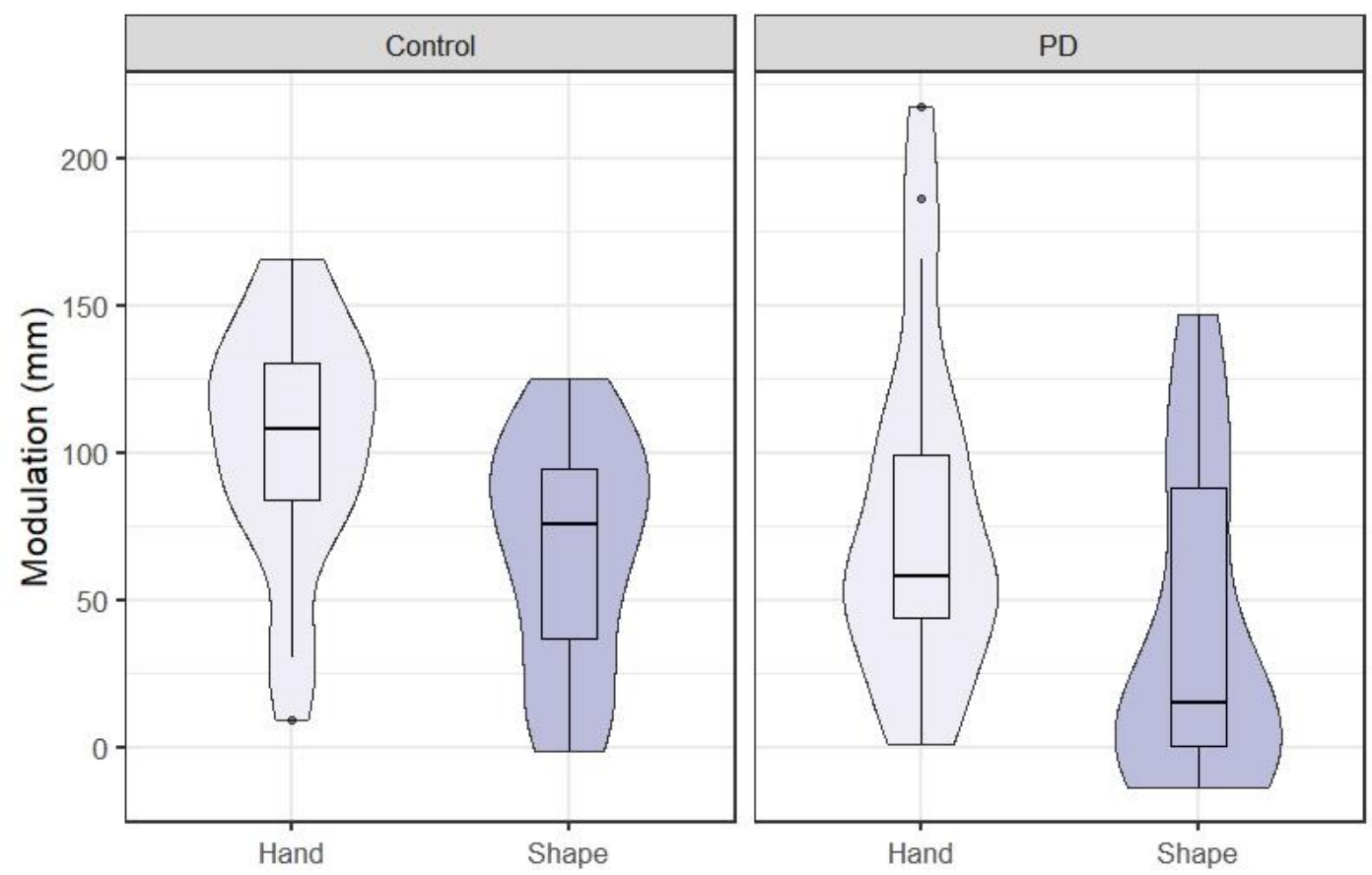

Figure 5. Modulation of vertical amplitude in response to changes in the trajectory of observed movements (elevated - direct) demonstrated by a human hand or simple shape stimulus. 
The effect of Group was not significant $\left(F(1,40)=3.92 ; p=.054 ; \eta^{2} p=.089 ; B_{10}=.39\right)$, but there was a significant interaction between Group and Trajectory $\left(F(1,40)=4.16 ; p=.048 ; \eta^{2} p=.094 ; B F_{\text {incl }}=6.60\right)$, reflecting greater overall modulation of vertical amplitude in the control group $(M=91.17 \mathrm{~mm})$ than the PD group $(\mathrm{M}=61.67 \mathrm{~mm}) ; \mathrm{t}(41)=2.03, \mathrm{p}=.049$. However, both groups exhibited significant modulation (control, $\mathrm{t}(40)=9.25 ; \mathrm{p}<.001 ; \mathrm{PD}, \mathrm{t}(40)=6.13 ; \mathrm{p}<.001)$. Interactions between Group and Stimulus $\left(\mathrm{F}(1,40)=.77 ; \mathrm{p}=.39 ; \eta^{2} p=.019 ; \mathrm{BF}_{\text {incl }}=0.28\right)$ and Group, Stimulus and Trajectory $\left(\mathrm{F}(1,40)=.14 ; \mathrm{p}=.71 ; \eta^{2} p\right.$ $=.003 ; \mathrm{BF}_{\text {incl }}=0.31$ ) were not significant.

\subsubsection{Eye movements}

Eye movement characteristics are summarised in Figure 6.

There was a significant effect of Stimulus on the number of fixations $\left(F(1,32)=13.27 ; p=.001 ; \eta^{2} p=.29\right.$; $\left.B F_{10}=42.87\right)$, which was higher when observing the Shape $(M=10.57)$ than the Hand $(M=9.61)$. There was no significant effect of Group $\left(\mathrm{F}(1,32)=.82 \mathrm{p}=.37 ; \eta^{2} p=.025 ; \mathrm{BF}_{10}=.61\right)$ or Stimulus $\times$ Group interaction $\left(F(1,32)=.096 ; p=.76 ; \eta^{2} p=.003 ; \mathrm{BF}_{\text {incl }}=.35\right)$. For fixation duration, there was again a significant effect of Stimulus $\left(F(1,32)=9.72 ; p=.004 ; \eta^{2} p=.023 ; \mathrm{BF}_{10}=10.73\right)$, with longer fixations for the Hand $(M=400.39 \mathrm{~ms})$ than the Shape $(M=355.73 \mathrm{~ms})$. There was no main effect of $\operatorname{Group}(F(1,32)=2.69$; $\left.\mathrm{p}=.11 ; \eta^{2} p=.077 ; \mathrm{BF}_{10}=1.18\right)$, or Stimulus $\times$ Group interaction $\left(\mathrm{F}(1,32)=.065 ; \mathrm{p}=.80 ; \eta^{2} p=.002 ; \mathrm{BF}_{\text {incl }}=\right.$ $.35)$.

Participants made a significantly larger number of saccades during the Shape $(M=9.85)$ than the Hand $(M=$ 8.92) block $\left(F(1,32)=10.85 ; p=.002 ; \eta^{2} p=.25 ; B_{10}=19.27\right)$. There was no significant effect of Group $\left(F(1,32)=1.81 ; p=.29 ; \eta^{2} p=.036 ; B_{10}=.67\right)$, or interaction between Stimulus and Group $(F(1,32)=.087 ; p$ $\left.=.77 ; \eta^{2} p=.003 ; \mathrm{BF}_{\text {incl }}=.34\right)$ on the number of saccades. Saccade amplitude was also larger in the Shape $(M=3.45)$ than the Hand $(M=3.18)$ block $\left(F(1,32)=6.92 ; p=.013 ; \eta^{2} p=.18 ; \mathrm{BF}_{10}=5.87\right)$. There was no significant effect of group on saccade amplitude $\left(\mathrm{F}(1,32)=.27 ; \mathrm{p}=.61 ; \eta^{2} p=.008 ; \mathrm{BF}_{10}=.43\right)$, or interaction between Stimulus and $\operatorname{Group}\left(F(1,32)=1.83 ; \mathrm{p}=.19 ; \eta^{2} p=.054 ; \mathrm{BF}_{\text {incl }}=.70\right)$.

The time of first fixation in the area around the movement endpoint differed significantly between stimuli $\left(F(1,32)=40.50 ; p<.001 ; \eta^{2} p=.56 ; B_{10}=1.15 e+6\right)$, reflecting earlier fixations with the Hand $(M=1296.56$ $\mathrm{ms})$ than the Shape $(\mathrm{M}=1537.22 \mathrm{~ms})$. There was no significant effect of Group $\left(\mathrm{F}(1,32)=1.28 ; \mathrm{p}=.27 ; \eta^{2} p\right.$ $\left.=.038 ; \mathrm{BF}_{10}=.39\right)$ or Group $\times$ Stimulus interaction $\left(\mathrm{F}(1,32)=.16 ; \mathrm{p}=.69 ; \eta^{2} p=.005 ; \mathrm{BF}_{\text {incl }}=.35\right)$. 

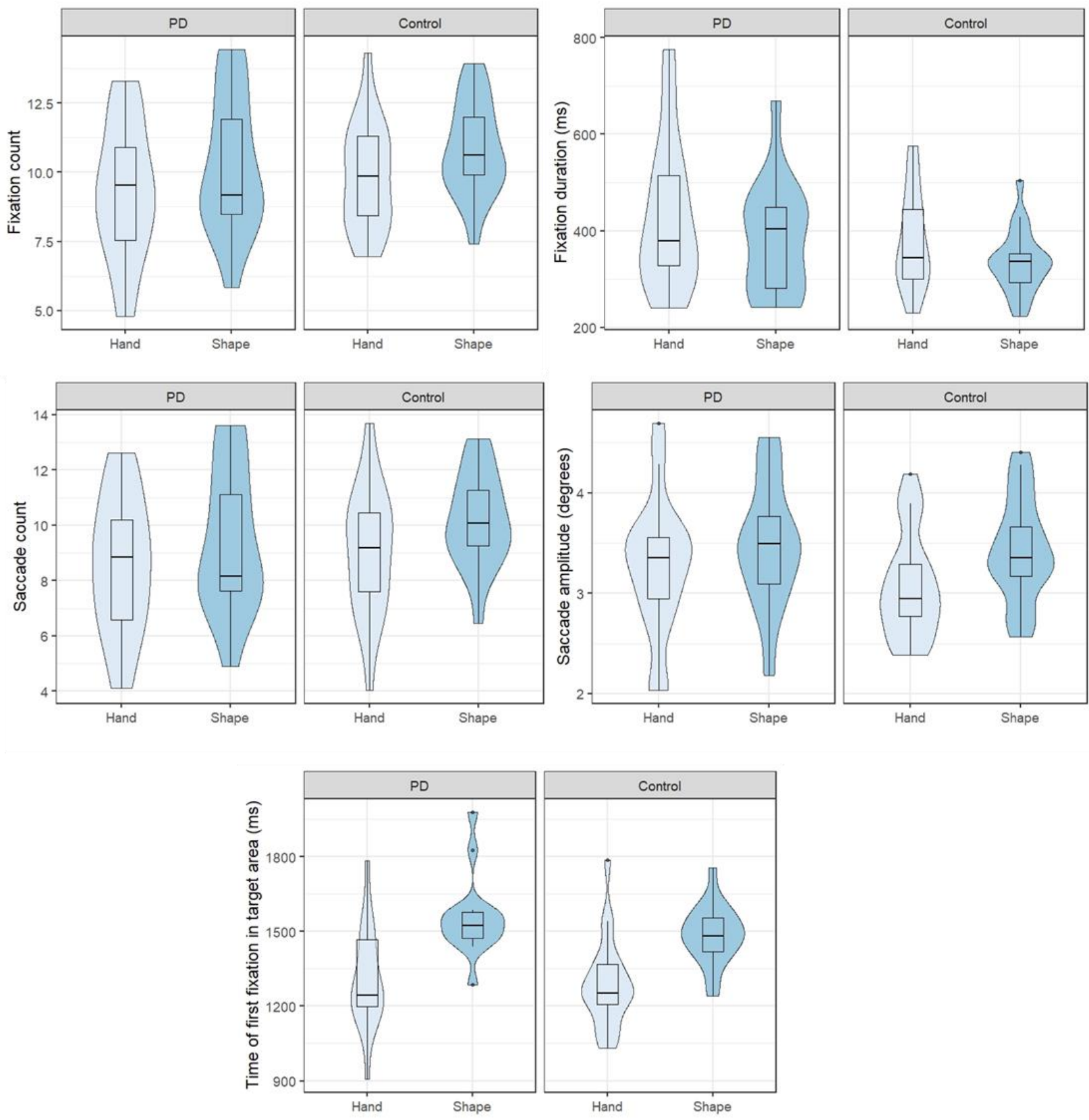

Figure 6. Eye movement characteristics (top to bottom panels: overall fixations; overall saccades; time of first fixation in the target area around the movement endpoint) when observing movements demonstrated by a human hand or simple shape stimulus prior to imitation.

\subsection{Discussion}

Experiment 2 showed that, in people with mild to moderate PD and age-matched controls, imitation of movement trajectory was increased when the movement was demonstrated by a human hand compared to a simple shape, despite the different stimuli having the same biological kinematic profile. Participants made larger movements in response to the human hand than the shape, and modulated the vertical 
amplitude of their own hand movements to a greater extent. As in Experiment 1, despite the PD group exhibiting smaller movements overall and showing some evidence of reduced modulation, a similar pattern of imitation was found across groups.

In both groups eye movements were also influenced by the type of stimulus observed: when watching the hand, participants exhibited fewer eye movements, longer fixations and smaller saccades than when watching the shape. This suggests that participants were tracking the hand more closely using smooth eye movements (classified as fixations), so requiring fewer and shorter saccades to follow the moving stimulus. Analysis of interest areas revealed that participants also fixated the movement endpoint earlier when watching the hand than the shape, suggesting increased prediction for biological movements.

Since both stimuli shared the same biological kinematics, the above differences in imitation and eye movements indicate that the visual characteristics of the stimulus are important for imitation in people with PD, consistent with the broader literature (see Gowen and Poliakoff, 2012). As hypothesised, movements demonstrated by a human agent may activate the action observation system more strongly than those with a non-biological appearance.

In the present study, participants made higher amplitude movements when imitating the human hand than the shape. This contrasts with the findings of McDonough et al. (2019), where participants underestimated the trajectory of an unobstructed elevated human reaching movement but not a non-biological equivalent. However, the movement in our task was not object-directed, and in both cases behaviour can be interpreted in relation to goal-directed accounts of imitation (e.g., Bekkering et al., 2000): when a visible movement goal is present, the observer's attention is focused on this as the intended endpoint of the action, whereas in the absence of such a cue the movement itself becomes the focus (see also Wild et al. 2012).

Alternatively, the difference could be accounted for by the greater visual salience of the hand relative to the shape, via increased attention to the movement kinematics. The difficulty in adequately matching biological and non-biological movement stimuli is acknowledged in the literature (Miller and Saygin, 2013); however, future research could attempt to match these more closely, such as by superimposing the shape on a larger background object.

\section{General discussion}

The present findings indicate greater effects of observing human movement than non-biological cues on imitation of hand movements, in terms of timing (Experiment 1) and trajectory (Experiment 2). In Experiment 1, observation of a human movement increased imitation of the duration, whereas imitation of horizontal amplitude was greater with a simple sequentially-presented non-biological cue. Moreover, viewing the sequence again during action execution (concurrent imitation) produced differential effects, increasing imitation of duration, but reducing imitation of amplitude. In Experiment 2, increased imitation of the movement trajectory and closer visual tracking were found for a human hand, compared to a simple moving shape with the same kinematic profile. Importantly, across the two experiments, people with PD imitated the movements of both biological and non-biological stimuli, exhibiting a similar pattern to an agematched control group.

These findings fit with a growing body of work demonstrating intact imitation in PD, at least at the mild to moderate stages, despite overall changes to the speed and amplitude of their movements and the potential 
involvement of the basal ganglia in action observation. A key contribution of the current work is that, unlike previous studies in PD, imitation of human movements and non-biological stimuli were compared, with the aim of increasing understanding of the underlying mechanisms. In conjunction with the authors' previous study demonstrating motor resonance for human movement in PD (Bek et al., 2018), these findings suggest that the route by which action observation activates sensorimotor processes is preserved in people with PD (Caligiore et al., 2017). This role of motor simulation during action observation in PD is further supported by the recent finding that deliberate engagement in motor imagery increased the effects of action observation on hand movement amplitude (Bek et al., 2019).

However, it should be acknowledged that the stronger effect of observing human action on imitation of movement kinematics in Experiment 2 could also be accounted for by the salience of the hand relative to the non-biological stimulus, which may have increased attention to the biological stimulus. Nonetheless, analysis of eye gaze suggested closer tracking and prediction of the ongoing movement of the hand than the shape. Together with the evidence outlined above, this suggests that motor simulation is the most parsimonious account of imitation effects in PD.

Despite the similar pattern of effects between groups, it is noted that the PD group exhibited reduced imitation overall relative to controls in Experiment 2, suggesting that further investigation is warranted. An important question for future research is whether people with PD would show effective imitation of human movement when tested off their dopaminergic medication. Moreover, the effects of tailoring instructions to increase motor imagery or visual attention could be investigated for both imitation and eye movements. In previous work attention and imagery instruction have been found to increase imitation of hand movements in healthy young adults (Bek et al., 2016; Hayes et al., 2014), and instructions to engage in motor imagery during observation increased imitation in healthy older adults and people with PD (Bek et al., 2019).

Although it is important to clarify the underlying mechanisms of imitation and cueing, the present findings nonetheless support the use of action observation as a potentially effective tool for neurorehabilitation in PD (see Abbruzzese et al., 2015; Caligiore et al., 2017). Moreover, AO could also be used to improve hand movements in healthy older adults, in whom manual dexterity can be compromised (e.g., Martin et al., 2015). AO-based strategies could be implemented flexibly within physiotherapy or home-based programmes, or following appropriate training could provide individuals with skills to apply independently to everyday functional tasks.

The present findings also indicate that different types and timing of stimuli may be useful in facilitating movement in accordance with the desired outcome, such as increasing speed or improving timing. In Experiment 1, modulation of movement duration was increased with action observation, but when simple cues were observed alone, duration was shorter overall and horizontal amplitude was imitated to a greater extent, possibly because attention was drawn to the positions of endpoint locations rather than the timing. Thus, while human action observation may be particularly effective in influencing movement kinematics or muscle activation (Hofree et al., 2015), simple cues may be effective when they provide sufficient information about a movement goal (e.g., a target endpoint for reaching). This is of relevance to the field of observational learning more broadly, as well as in rehabilitation, whereby training could focus on a particular aspect of movement (see Bienkiewicz et al., 2013; Nackaerts et al., 2017). If the aim is to move the effector to a particular location or to complete a movement as quickly as possible, simple visual cues may be the optimal choice, whereas if precision of timing or trajectory is to be improved, observation of human movement may be more effective. Importantly, action observation could also be more readily 
applied to activities of daily living, including those requiring finer motor skills - such as complex manual tasks - that could not be adequately guided by simple cues. Experiment 1 additionally indicated that presenting stimuli online during action execution may increase the effects on movement duration (cf. Krüger et al., 2014), compared to sequential observation and imitation.

In conclusion, people with mild to moderate PD, despite producing smaller and slower hand movements than age-matched controls, nevertheless exhibited similar patterns of imitation across stimulus conditions. Future research should further investigate the mechanisms underlying imitation of biological and nonbiological stimuli in ageing and neurodegenerative conditions.

\section{Acknowledgements}

The authors thank Dr Matthew Sullivan and Dr Emma Stack for their input into the design of these studies and Dr Jeremy Dick for assistance with participant recruitment. We would also like to thank all the participants involved in the study.

\section{Funding}

This work was supported by the Economic and Social Research Council [Grant Number ES/K013564/1].

\section{$\underline{\text { References }}$}

Abbruzzese, G., Avanzino, L., Marchese, R., Pelosin, E., 2015. Action Observation and Motor Imagery: Innovative Cognitive Tools in the Rehabilitation of Parkinson's Disease. Parkinsons. Dis. https://doi.org/10.1155/2015/124214

Agosta, F., Gatti, R., Sarasso, E., Volonte, M.A., Canu, E., Meani, A., Sarro, L., Copetti, M., Cattrysse, E., Kerckhofs, E., Comi, G., Falini, A., Filippi, M., 2017. Brain plasticity in Parkinson's disease with freezing of gait induced by action observation training. J. Neurol. 264, 88-101. https://doi.org/10.1007/s00415-016-8309-7

Alegre, M., Guridi, J., Artieda, J., 2011. The mirror system, theory of mind and Parkinson's disease. J. Neurol. Sci. 310, 194-196. https://doi.org/10.1016/j.jns.2011.07.030

Alegre, M., Rodriguez-Oroz, M.C., Valencia, M., Perez-Alcazar, M., Guridi, J., Iriarte, J., Obeso, J.A., Artieda, J., 2010. Changes in subthalamic activity during movement observation in Parkinson's disease: Is the mirror system mirrored in the basal ganglia? Clin. Neurophysiol. 121, 414-425. https://doi.org/10.1016/j.clinph.2009.11.013

Bek, J., Gowen, E., Vogt, S., Crawford, T., Poliakoff, E., 2018. Action observation produces motor resonance in Parkinson's disease. J. Neuropsychol. 12, 298-311. https://doi.org/10.1111/jnp.12133

Bek, J., Gowen, E., Vogt, S., Crawford, T.J., Poliakoff, E., 2019. Combined action observation and motor imagery influences hand movement amplitude in Parkinson's disease. Park. Relat. Disord. https://doi.org/10.1016/j.parkreldis.2018.11.001

Bek, J., Poliakoff, E., Marshall, H., Trueman, S., Gowen, E., 2016. Enhancing voluntary imitation through attention and motor imagery. Exp. Brain Res. 234. https://doi.org/10.1007/s00221-016-4570-3

Bekkering, H., Wohlschlager, A., Gattis, M., 2000. Imitation of gestures in children is goal-directed. Q. J. Exp. Psychol. Sect. a-Human Exp. Psychol. 53, 153-164. https://doi.org/10.1080/027249800390718 
Bienkiewicz, M.M.N., Rodger, M.W.M., Young, W.R., Craig, C.M., 2013. Time to get a move on: Overcoming bradykinetic movement in Parkinson's disease with artificial sensory guidance generated from biological motion. Behav. Brain Res. 253, 113-120. https://doi.org/10.1016/j.bbr.2013.07.003

Bonivento, C., Rumiati, R.I., Biasutti, E., Humphreys, G.W., 2013. The role of the basal ganglia in action imitation: neuropsychological evidence from Parkinson's disease patients. Exp. Brain Res. 224, 211220. https://doi.org/10.1007/s00221-012-3300-8

Borges, L.R., Fernandes, A.B., Melo, L.P., Guerra, R.O., Campos, T.F., 2018. Action observation for upper limb rehabilitation after stroke. Cochrane database Syst. Rev. https://doi.org/10.1002/14651858.CD011887.pub2

Brown, R.G., Marsden, C.D., 1988. INTERNAL VERSUS EXTERNAL CUES AND THE CONTROL OF ATTENTION IN PARKINSONS-DISEASE. Brain 111, 323-345. https://doi.org/10.1093/brain/111.2.323

Buccino, G., 2014. Action observation treatment: a novel tool in neurorehabilitation. Philos. Trans. R. Soc. B-Biological Sci. 369. https://doi.org/10.1098/rstb.2013.0185

Buccino, G., Gatti, R., Giusti, M.C., Negrotti, A., Rossi, A., Calzetti, S., Cappa, S.F., 2011. Action observation treatment improves autonomy in daily activities in Parkinson's disease patients: Results from a pilot study. Mov. Disord. https://doi.org/10.1002/mds.23745

Caligiore, D., Mustile, M., Spalletta, G., Baldassarre, G., 2017. Action observation and motor imagery for rehabilitation in Parkinson's disease: A systematic review and an integrative hypothesis. Neurosci. Biobehav. Rev. 72, 210-222. https://doi.org/10.1016/j.neubiorev.2016.11.005

Caligiore, D., Pezzulo, G., Miall, R.C., Baldassarre, G., 2013. The contribution of brain sub-cortical loops in the expression and acquisition of action understanding abilities. Neurosci. Biobehav. Rev. 37, 25042515. https://doi.org/10.1016/j.neubiorev.2013.07.016

Caspers, S., Zilles, K., Laird, A.R., Eickhoff, S.B., 2010. ALE meta-analysis of action observation and imitation in the human brain. Neuroimage 50, 1148-1167. https://doi.org/10.1016/j.neuroimage.2009.12.112

Castiello, U., Ansuini, C., Bulgheroni, M., Scaravilli, T., Nicoletti, R., 2009. Visuomotor priming effects in Parkinson's disease patients depend on the match between the observed and the executed action. Neuropsychologia 47, 835-842. https://doi.org/10.1016/j.neuropsychologia.2008.12.016

Craighero, L., Jacono, M., Mele, S., 2016. Resonating with the ghost of a hand: A TMS experiment. Neuropsychologia. https://doi.org/10.1016/j.neuropsychologia.2016.02.014

Crescentini, C., Mengotti, P., Grecucci, A., Rumiati, R.I., 2011. The effect of observed biological and non biological movements on action imitation: An fMRI study. Brain Res.

https://doi.org/10.1016/j.brainres.2011.08.077

Eaves, D.L., Riach, M., Holmes, P.S., Wright, D.J., 2016. Motor Imagery during Action Observation: A Brief Review of Evidence, Theory and Future Research Opportunities. Front. Neurosci. 10. https://doi.org/10.3382/fnins.2015.00514

Ertelt, D., Small, S., Solodkin, A., Dettmers, C., McNamara, A., Binkofski, F., Buccino, G., 2007. Action observation has a positive impact on rehabilitation of motor deficits after stroke. Neuroimage 36, T164-T173. https://doi.org/10.1016/j.neuroimage.2007.03.043

Giorgi, G., Ferrarello, F., Merlo, F., Fumagalli, S., Marchionni, N., Di Bari, M., 2018. First-Person Perspective Action Observation Training in Individuals With Parkinson's Disease. J. Geriatr. Phys. Ther. https://doi.org/10.1519/jpt.0000000000000113 
Gowen, E., Poliakoff, E., 2012. How does visuomotor priming differ for biological and non-biological stimuli? A review of the evidence. Psychol. Res. Forsch. 76, 407-420. https://doi.org/10.1007/s00426-0110389-5

Hardwick, R.M., Caspers, S., Eickhoff, S.B., Swinnen, S.P., 2018. Neural correlates of action: Comparing meta-analyses of imagery, observation, and execution. Neurosci. Biobehav. Rev. https://doi.org/10.1016/j.neubiorev.2018.08.003

Haslinger, B., Erhard, P., Altenmüller, E., Schroeder, U., Boecker, H., Ceballos-Baumann, A.O., 2005. Transmodal sensorimotor networks during action observation in professional pianists. J. Cogn. Neurosci. https://doi.org/10.1162/0898929053124893

Hayes, S.J., Roberts, J.W., Elliott, D., Bennett, S.J., 2014. Top-Down Attentional Processes Modulate the Coding of Atypical Biological Motion Kinematics in the Absence of Motor Signals. J. Exp. Psychol. Percept. Perform. 40, 1641-1653. https://doi.org/10.1037/a0037200

Helmich, R.C., Bloem, B.R., Toni, I., 2012. Motor imagery evokes increased somatosensory activity in parkinson's disease patients with tremor. Hum. Brain Mapp. 33, 1763-1779. https://doi.org/10.1002/hbm.21318

Helmich, R.C., de Lange, F.P., Bloem, B.R., Toni, I., 2007. Cerebral compensation during motor imagery in Parkinson's disease. Neuropsychologia 45, 2201-2215. https://doi.org/10.1016/j.neuropsychologia.2007.02.024

Higuchi, S., Holle, H., Roberts, N., Eickhoff, S.B., Vogt, S., 2012. Imitation and observational learning of hand actions: Prefrontal involvement and connectivity. Neuroimage 59, 1668-1683. https://doi.org/10.1016/j.neuroimage.2011.09.021

Hofree, G., Urgen, B.A., Winkielman, P., Saygin, A.P., 2015. Observation and imitation of actions performed by humans, androids, and robots: An EMG study. Front. Hum. Neurosci. https://doi.org/10.3389/fnhum.2015.00364

Holmes, P., Calmels, C., 2008. A neuroscientific review of imagery and observation use in sport. J. Mot. Behav. https://doi.org/10.3200/JMBR.40.5.433-445

Hsieh, S., Schubert, S., Hoon, C., Mioshi, E., Hodges, J.R., 2013. Validation of the Addenbrooke's Cognitive Examination III in Frontotemporal Dementia and Alzheimer's Disease. Dement. Geriatr. Cogn. Disord. 36, 242-250. https://doi.org/10.1159/000351671

lacoboni, M., Molnar-Szakacs, I., Gallese, V., Buccino, G., Mazziotta, J.C., Rizzolatti, G., 2005. Grasping the intentions of others with one's own mirror neuron system. Plos Biol. 3, 529-535. https://doi.org/10.1371/journal.pbio.0030079

Jaywant, A., Ellis, T.D., Roy, S., Lin, C.-C., Neargarder, S., Cronin-Golomb, A., 2016a. Randomized Controlled Trial of a Home-Based Action Observation Intervention to Improve Walking in Parkinson Disease. Arch. Phys. Med. Rehabil. 97, 665-673. https://doi.org/10.1016/j.apmr.2015.12.029

Jaywant, A., Shiffrar, M., Roy, S., Cronin-Golomb, A., 2016b. Impaired perception of biological motion in parkinson's disease. Neuropsychology. https://doi.org/10.1037/neu0000276

Kessler, K., Biermann-Ruben, K., Jonas, M., Siebner, H.R., Baumer, T., Munchau, A., Schnitzler, A., 2006. Investigating the human mirror neuron system by means of cortical synchronization during the imitation of biological movements. Neuroimage 33, 227-238.

https://doi.org/10.1016/j.neuroimage.2006.06.014

Krüger, B., Bischoff, M., Blecker, C., Langhanns, C., Kindermann, S., Sauerbier, I., Reiser, M., Stark, R., 
Munzert, J., Pilgramm, S., 2014. Parietal and premotor cortices: Activation reflects imitation accuracy during observation, delayed imitation and concurrent imitation. Neuroimage.

https://doi.org/10.1016/j.neuroimage.2014.05.074

Leiguarda, R., 2001. Limb apraxia: Cortical or subcortical. Neuroimage 14, S137-S141. https://doi.org/10.1006/nimg.2001.0833

Leiguarda, R.C., Pramstaller, P.P., Merello, M., Starkstein, S., Lees, A.J., Marsden, C.D., 1997. Apraxia in Parkinson's disease, progressive supranuclear palsy, multiple system atrophy and neuroleptic-induced parkinsonism. Brain 120, 75-90. https://doi.org/10.1093/brain/120.1.75

Liu, T., Hu, P., Cao, R., Ye, X., Tian, Y., Chen, X., Wang, K., 2017. Dopaminergic Modulation of Biological Motion Perception in patients with Parkinson's disease. Sci. Rep. https://doi.org/10.1038/s41598-01710463-2

Martin, J.A., Ramsay, J., Hughes, C., Peters, D.M., Edwards, M.G., 2015. Age and grip strength predict hand dexterity in adults. PLoS One. https://doi.org/10.1371/journal.pone.0117598

McDonough, K.L., Hudson, M., Bach, P., 2019. Cues to intention bias action perception toward the most efficient trajectory. Sci. Rep. https://doi.org/10.1038/s41598-019-42204-y

Miller, L.E., Saygin, A.P., 2013. Individual differences in the perception of biological motion: Links to social cognition and motor imagery. Cognition. https://doi.org/10.1016/j.cognition.2013.03.013

Nackaerts, E., Broeder, S., Pereira, M.P., Swinnen, S.P., Vandenberghe, W., Nieuwboer, A., Heremans, E., 2017. Handwriting training in Parkinson's disease: A trade-off between size, speed and fluency. PLoS One. https://doi.org/10.1371/journal.pone.0190223

Pelosin, E., Bove, M., Ruggeri, P., Avanzino, L., Abbruzzese, G., 2013. Reduction of Bradykinesia of Finger Movements by a Single Session of Action Observation in Parkinson Disease. Neurorehabil. Neural Repair 27, 552-560. https://doi.org/10.1177/1545968312471905

Poliakoff, E., 2013. Representation of action in Parkinson's disease: Imagining, observing, and naming actions. J. Neuropsychol. 7, 241-254. https://doi.org/10.1111/jnp.12005

Redgrave, P., Rodriguez, M., Smith, Y., Rodriguez-Oroz, M.C., Lehericy, S., Bergman, H., Agid, Y., DeLong, M.R., Obeso, J.A., 2010. Goal-directed and habitual control in the basal ganglia: implications for Parkinson's disease. Nat. Rev. Neurosci. 11, 760-772. https://doi.org/10.1038/nrn2915

Ricciardi, L., Visco-Comandini, F., Erro, R., Morgante, F., Bologna, M., Fasano, A., Ricciardi, D., Edwards, M.J., Kilner, J., 2017. Facial emotion recognition and expression in Parkinson's disease: An emotional mirror mechanism? PLoS One. https://doi.org/10.1371/journal.pone.0169110

Robles-Garcia, V., Arias, P., Sanmartin, G., Espinosa, N., Flores, J., Grieve, K.L., Cudeiro, J., 2013. Motor facilitation during real-time movement imitation in Parkinson's disease: A virtual reality study. Parkinsonism Relat. Disord. 19, 1123-1129. https://doi.org/10.1016/j.parkreldis.2013.08.005

Sakreida, K., Higuchi, S., Di Dio, C., Ziessler, M., Turgeon, M., Roberts, N., Vogt, S., 2018. Cognitive control structures in the imitation learning of spatial sequences and rhythms - An fMRI study. Cereb. Cortex. https://doi.org/10.1093/cercor/bhw414

Simons, G., Smith Pasqualini, M.C., Reddy, V., Wood, J., 2004. Emotional and nonemotional facial expressions in people with Parkinson's disease. J. Int. Neuropsychol. Soc. https://doi.org/10.1017/S135561770410413X

Spaulding, S.J., Barber, B., Colby, M., Cormack, B., Mick, T., Jenkins, M.E., 2013. Cueing and Gait 
Improvement Among People With Parkinson's Disease: A Meta-Analysis. Arch. Phys. Med. Rehabil. 94, 562-570. https://doi.org/10.1016/j.apmr.2012.10.026

Stefan, K., Classen, J., Celnik, P., Cohen, L.G., 2008. Concurrent action observation modulates practiceinduced motor memory formation. Eur. J. Neurosci. 27, 730-738. https://doi.org/10.1111/j.14609568.2008.06035.x

van Nuenen, B.F.L., Helmich, R.C., Buenen, N., van de Warrenburg, B.P.C., Bloem, B.R., Toni, I., 2012. Compensatory Activity in the Extrastriate Body Area of Parkinson's Disease Patients. J. Neurosci. 32, 9546-9553. https://doi.org/10.1523/jneurosci.0335-12.2012

Van Selst, M., Jolicoeur, P., 1994. A Solution to the Effect of Sample Size on Outlier Elimination. Q. J. Exp. Psychol. Sect. A. https://doi.org/10.1080/14640749408401131

van Wegen, E.E.H., Hirsch, M.A., Huiskamp, M., Kwakkel, G., 2014. Harnessing Cueing Training for Neuroplasticity in Parkinson Disease. Top. Geriatr. Rehabil. 30, 46-57. https://doi.org/10.1097/tgr.0000000000000005

Vogt, S., Thomaschke, R., 2007. From visuo-motor interactions to imitation learning: Behavioural and brain imaging studies. J. Sports Sci. 25, 497-517. https://doi.org/10.1080/02640410600946779

Wagenmakers, E.J., Love, J., Marsman, M., Jamil, T., Ly, A., Verhagen, J., Selker, R., Gronau, Q.F., Dropmann, D., Boutin, B., Meerhoff, F., Knight, P., Raj, A., van Kesteren, E.J., van Doorn, J., Šmíra, M., Epskamp, S., Etz, A., Matzke, D., de Jong, T., van den Bergh, D., Sarafoglou, A., Steingroever, H., Derks, K., Rouder, J.N., Morey, R.D., 2018. Bayesian inference for psychology. Part II: Example applications with JASP. Psychon. Bull. Rev. https://doi.org/10.3758/s13423-017-1323-7

Wild, K.S., Poliakoff, E., Jerrison, A., Gowen, E., 2012. Goal-Directed and Goal-Less Imitation in Autism Spectrum Disorder. J. Autism Dev. Disord. 42, 1739-1749. https://doi.org/10.1007/s10803-011-14174

Wild, K.S., Poliakoff, E., Jerrison, A., Gowen, E., 2010. The influence of goals on movement kinematics during imitation. Exp. Brain Res. 204, 353-360. https://doi.org/10.1007/s00221-009-2034-8 